\title{
NETWORKED READING
}

Dear Reader,

Things are really moving in the commercial vehicle industry. Not that this sector has been in any state of hibernation until now, but new legislation requires renewed efforts in exhaust emissions control and the introduction of driver assistance systems. Like its sister magazines ATZ and ATZelektronik, MTZ will reflect these developments and focus more strongly on commercial vehicles in the future - even beyond the main topics.

But this is by no means all of the common features shared by the three sister magazines. In future, we will pay more attention to examining topics across all three publications, and to interconnect the different perspectives in an optimum manner. For example, MTZ might take an in-depth look at a new engine, while ATZ examines its integration into the complete vehicle and ATZelektonik scrutinises the details of its electrics and electronics. And that's not all: there's plenty happening online too. Here, we will focus more intensively on technical road test reports and generally make sure that we are absolutely up to date. In addition, our new platform "Springer für Professionals" will soon not only be offering the familiar topics from ATZonline but will also provide outstanding research possibilities that cover the entire network of our specialist book publications and technical articles.

Like to know more? There's no need to wait until next year. Take a look at ATZ 12, which comes out shortly after MTZ. In the new issue, MAN presents a concept for improving the transport efficiency of $40 \mathrm{t}$ trucks, while the TU Braunschweig examines the modelbased analysis of safety-enhancing measures, illustrated by the design of modern driver assistance systems. It is also well worth taking a look at ATZelektronik 6, which is published in December. Topics in this issue include a detailed report on ISO 26262 for software development in commercial vehicles in relation to driver assistance systems from the point of view of MAN. Or you can study the software report by IAV on the self-calibration of ECUs.

Networked reading is an excellent idea. By not only reading MTZ but also looking at ATZ and ATZelektronik and of course making use of our online resources, you can be certain that you have the best possible access to interesting and important sources of information.

Best regards,

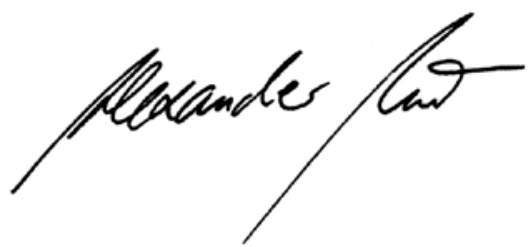

DR. ALEXANDER HEINTZEL, Editor in Chief Wiesbaden, 12 October 2012

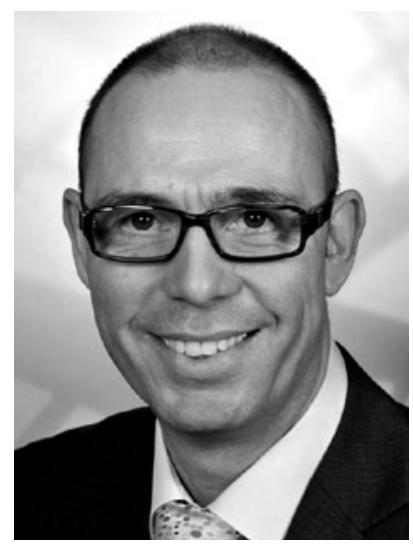

